Ann. Zootech., I969, 18 (2), I69-I84.

\title{
ÉTUDE PRÉLIMINAIRE DE LA PHASE ASCENDANTE DE LA COURBE DE LACTATION DES BREBIS TRAITES
}

\author{
J.-A. IZQUIERDO PRIMO ('), J.-C. FLAMANT et G. RICORDEAU \\ Station de Génétique quantitative et appliquée, \\ Centre national de Recherches zootechniques, 78 -Jouy-en-Josas \\ Institut national de la Recherche agronomique
}

\section{SOMMAIRE}

Sur un troupeau de brebis de race pure Préalpes et croisées Frisonnes $\left(\mathbf{F}_{1}\right.$ et $\left.3 / 4 F\right)$ on a étudié le stade d'apparition du maximum de la lactation.

Les distributions de fréquence de cette variable sont très dissymétriques et la transformation logarithmique ne permet pas un ajustement à la loi normale. Cet ajustement est réalisé en partie avec une transformation racine carrée. La dissymétrie constatée pourrait donc provenir du fait que la semaine d'apparition du maximum, variable bolnée inférieurement à o mais non supérieurement, s'applique à un événement de faible probabilité. Une autre explication possible, s'appuyant sur les caractéristiques des courbes de descente du lait au cours de la traite, n'est pas vérifiable avec l'ensemble de nos données.

Les principaux facteurs de variation observés sont le numéro de lactation et la race, les animaux en deuxième lactation et les animaux croisés ayant un maximum de lactation plus précoce. Après correction pour le numéro de lactation, la corrélation entre la durée de la phase ascendante et le niveau de production, calculée pour chaque race, reste faible et négative.

\section{INTRODUCTION}

La phase ascendante de la courbe de lactation des brebis traites n'a pas été étudiée de façon détaillée jusqu'à maintenant. De nombreux auteurs ont obtenu divers résultats quant à la position du maximum de la lactation en relation avec le niveau de production. Ces études portent en fait sur la quantité de lait consommée par l'agneau durant la période d'allaitement, quantité estimée par pesée avant et après la tétée. Or, comme le montrent les travaux de RiCORDEAU et DENAMUR (I962), LABUSSIÈRE et PETREQUiN (I969), la quantité de lait produite par une brebis pen-

(1) Adresse actuelle : Jefatura, agronomica Leon (Espagne). 
dant la période d'allaitement dépend du nombre d'agneaux allaités et du stimulus lié à la présence de l'agneau : elle risque donc de constituer une mauvaise estimation de la quantité de lait obtenue avec la même brebis à la traite.

Dans les conditions habituelles d'élevage en ferme de la zone de Roquefort (race Lacaune), les agneaux sont séparés de leur mère après 30 à 40 jours d'allaitement : la période de traite débute donc tardivement. Fin outre, l'interruption brutale de la tétée des agneaux ne permet pas d'obtenir immédiatement une traite normale : LABUSSIÈre et PeTrequn (r969) ont montré que dans ce cas, le réflexe d'éjection était presque toujours absent et qu'il fallait attendre 2 à 3 semaines de traite quotidienne pour le voir s'établir. Si l'on veut étudier l'évolution de la production laitière journalière des brebis depuis l'agnelage jusqu'au maximum de production, il est donc indispensable de disposer de contrôles laitiers fréquents réalisés sur des brebis soumises à la traite à la fin de la période colostrale. C'est la raison pour laquelle cette étude porte, non pas sur les données du contrôle laitier de la région de Roquefort, mais sur des observations recueillies en station, les brebis étant maintenues en stabulation pendant toute la période de lactation.

\section{MATÉRIEL ET MÉTHODES}

I. Des mesures quotidiennes de productions laitières individuelles ont été effectuées durant 4 années, à la Ferme expérimentale de Brouessy, sur un troupeau constitué à l'occasion d'une expérience de croisement entre brebis Préalpes $d u$ Sud et béliers Firisons (Ostfriesisches Milchschaf). Le déroulement de cette expérimentation et ses résultats ont été décrits et discutés par RicoRDEAU et Flamant $(\mathrm{Ig} 69, a, b)$. Les données prises ici en considération sont relatives aux brebis de race pure Préalpes du Sud, aux produits de croisements de première génération $\mathrm{F}_{1}(\widehat{\partial} \widehat{o}$ Frison $\times$ o 0 Préalpes $)$ et aux croisements en retour $3 / 4 \mathrm{~F}\left(\widehat{\partial} \widehat{\delta}\right.$ Frison $\times \propto q \mathrm{~F}_{1}$; cette catégorie contient également quelques animaux $5 / 8$ et $7 / 8$ de sang Frison).

2. Après avoir éliminé les lactations de durée anormalement courte (moins de 60 jours), nous avons retenu pour notre étude 544 lactations de 282 brebis. La totalité des mises bas est intervenue approximativement dans un intervalle de 2 mois pour chaque campagne, les toutes premières mises bas s'effectuant à la fin de décembre et les dernières au début de mars, la plus grande partie en janvier. Les brebis ont mis bas la première fois à l'âge de un an, sauf 2 lots qui ont eu leur premier agnelage à 2 ans : ceux-ci ont été considérés uniquement pour l'étude de l'influence de l'âge à la première mise bas. Les agneaux ont été séparés de leur mère après avoir tété le lait colostral, soit 4 jours au plus tard après leur naissance.

3. La traite a été effectuée 2 fois par jour en 2 opérations: une traite-machine suivie d'un égouttage manuel après enlèvement des gobelets. La quantité de lait obtenue au cours de chacune de ces 2 opérations a été mesurée quotidiennement matin et soir. Nous avons calculé la production totale journalière en additionnant les 4 données d'une journée. Les critères de production suivants ont été retenus pour notre analyse : niveau maximum pour la traite du matin (machine-matin), niveau maximum pour la traite du soir (machine-soir), niveau maximum total pour les 2 traitesquotidiennes.

La durée de la phase ascendante de la lactation a été déterminée d'après le jour d'apparition, par rapport à la date d'agnelage, de la production maximum pour les 3 critères cités. Si le maximum persistait plusieurs jours de suite, nous avons retenu le premier jour seulement. DECAEN et Poutous (I965) ont en effet montré sur les bovins laitiers que le regroupement des productions par période de 5 jours ne permet pas d'apprécier avec plus de précision la position du maximum de la lactation.

4. L'étude de la distribution de fréquence de chacune des variables a été réalisée par la détermination des coefficients étudiés par GEARY et PEARSON : $a, \sqrt{b_{1}}$ et $b_{2}$.

$a=$ Indice d'aplatissement des distributions d'effectif supérieur à to : déviation moyenne/écart-type

$b_{\mathbf{2}}=$ Indice d'aplatissement des distributions d'effectif supérieur à I $99: \frac{m_{4}}{m_{2}^{2}}$

$\dot{\sqrt{b_{1}}}=$ Indice de dissymétrie $: \frac{m_{3}}{m_{2}{ }^{3 / 2}}$ 
$m_{1}, m_{2}, m_{3}$ et $m_{4}$ étant respectivement les moments de $\mathrm{I}^{\mathrm{er}}, 2^{\mathrm{e}}, 3^{\mathrm{e}}$ et $4^{\mathrm{e}}$ ordre de la distribution.

Pour les tests de normalité (tabl. 2), les valeurs calculées ont été comparées aux valeurs théoricpues d'après les tables de Pearson et HartLey (1958).

5. Du fait du déroulement dans le temps du programme de croisement, l’étude des effets des différents facteurs de variation (âge à la première mise bas, année, race) a porté sur des schémas non balancés. Pour pallier en partie cet inconvénient, l'analyse de la variance a été réalisée sur des lots d'animaux comparables : mise bas :

- de même race et de même numéro de lactation pour étudier l'effet de l'âge à la première

- de même âge et de même année de production pour étudier l'effet de la race ;

- de même race et de même âge pour étudier l'effet de l'année de production ;

- de même année de production et de même race pour étudier l'effet de l'âge des brebis.

Une analyse de variance hiérarchique considérant les facteurs de classification "race " et "brebis" a permis d'étudier l'importance du facteur brebis sur les caractéristiques de la courbe ascendante de la lactation ainsi que les coefficients de corrélation entre les différents critères considérés. l'ensemble des calculs a été réalisé sur les données non transformées.

\section{RÉSULTATS}

\section{Distributions de fréquence}

En moyenne, le maximum de la lactation intervient au début de la $4^{\mathrm{e}}$ semaine, les extrêmes étant 2 et 74 jours ; la plus grande fréquence est observée au cours de la $3^{\text {e }}$ semaine. En effet, la durée de la phase ascendante, et également la production maximum, ont une distribution dissymétrique : pour chaque race ou pour l'ensemble des données, les coefficients $\sqrt{b_{1}}$ sont très significatifs. La dissymétrie est surtout accentuée pour la durée de la phase ascendante avec un étalement des distributions vers les valeurs élevées (tab1. I, fig. I). Les écarts par rapport à une distribution nor-

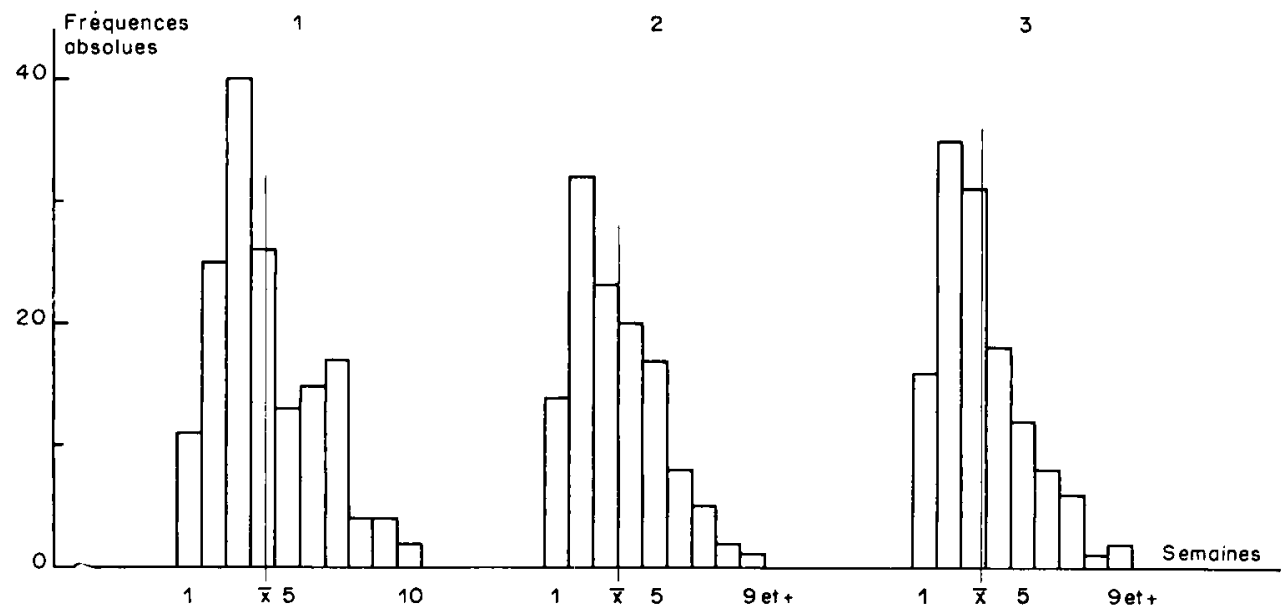

1G. 1. - Distributions de tréquence de la durée de la phase ascendante de la laclation
I. Préalpes.
2. $\mathrm{F}_{1}$.
3. $3 / 4 \mathrm{~F}$

male sont beaucoup moins importants pour l'indice d'aplatissement (kurtosis) et on peut admettre qu'il n'y a pas de différences pour ce caractère. La transformation 


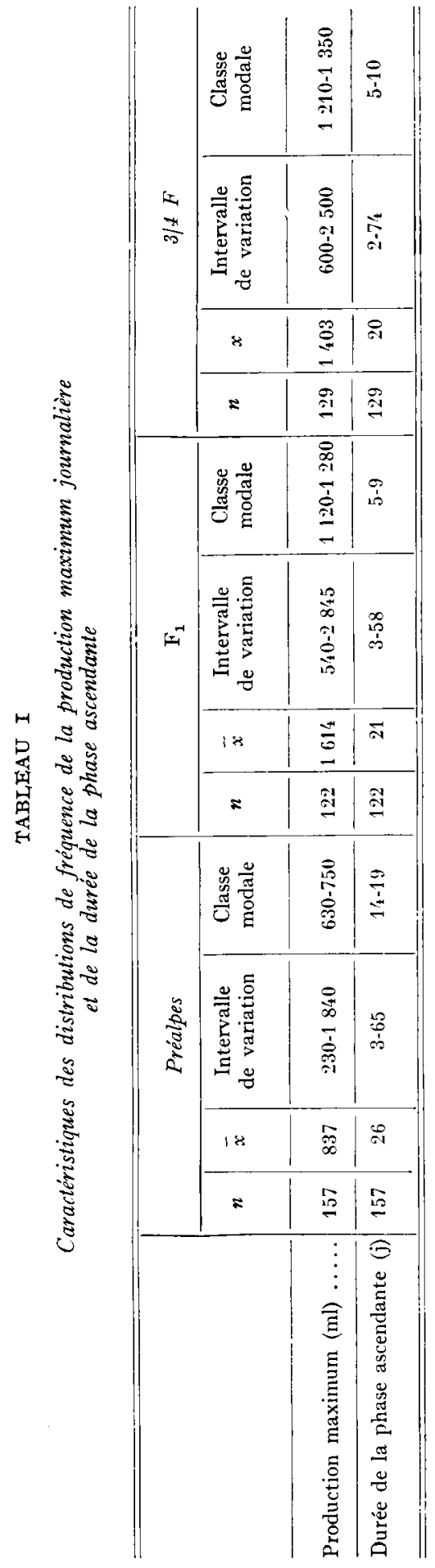


COURBE DE IACTATION DES BREBIS TRAITES

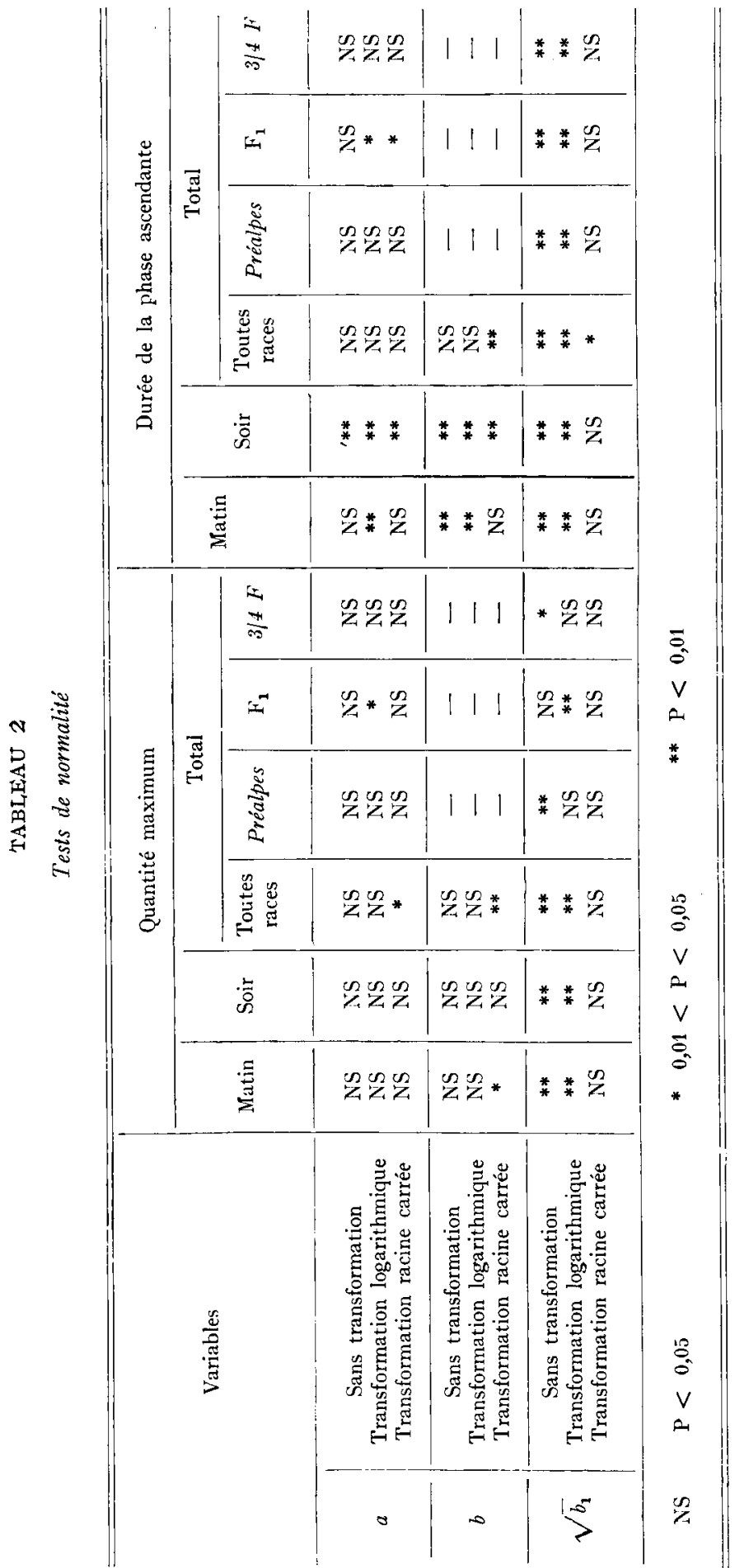


des variables selon une échelle logarithmique ne permet pas d'obtenir des distributions symétriques. Par contre, les distributions des racines carrées des données pour toutes les variables sont symétriques mais avec une tendance à l'aplatissement (tests $a$ et $b_{2}$ significatifs dans presque $5^{\circ}$ p. Ioo des cas, tab1. 2).

Pour la production maximum, les coefficients de variation sont compris entre 25 et $35 \mathrm{p}$. Ioo et sont comparables à ceux généralement obtenus pour la production totale par lactation chez les Bovins (DECAEN et Poutous, I965) et chez les Ovins (Boyazoglu et CatTin-VidaI, I964). Le coefficient de variation de la durée de la phase ascendante est beaucoup plus élevé (6o p. I00) : cette variabilité importante provient du nombre non négligeable de lactations qui ont une phase ascendante très longue.

\section{Facteurs de variation de la production maximum}

Nous considérons globalement les 3 variables étudiées pour lesquelles les résultats des analyses ont été identiques et qui présentent entre elles une liaison très étroite : les coefficients de corrélation varient de 0,79 à 0,96 (tabl. 3).

\section{TABLEAU 3}

Coefficients de corrélation intra-brebis entre les différentes variables

\begin{tabular}{|c|c|c|c|c|c|}
\hline & Préalpes & $\mathrm{F}_{1}$ & $3 / 4 F$ & Tous & $s$ animaux \\
\hline Maximum matin et Maximum soir .. & $0,79^{* *}$ & $0,90 * *$ & 0,$8 ; * *$ & & $0,86 * *$ \\
\hline Maximum matin et Maximum total. & $0,90^{* * *}$ & $0,96 * *$ & $0,90 * *$ & & $0,94 * *$ \\
\hline Maximum soir et Maximum total ... & $0,91^{* *}$ & $0,95 * *$ & $0,91 * *$ & & $0,93^{* *}$ \\
\hline Durée matin et Durée soir ........ & $0,45^{* *}$ & $0,59 * *$ & $0,38^{* *}$ & & $0,50 * *$ \\
\hline Durée matin et Durée totale ....... & $0,78^{* *}$ & $0,85 * *$ & $0,54 * *$ & & $0,77 * *$ \\
\hline Durée soir et Durce totale ........ & $0,59 * *$ & $0,73^{* *}$ & $0,72 * *$ & & $0,68 * *$ \\
\hline Maximum matin et Duréc matin .... & $-0,79 * *$ & $\longrightarrow 0,5.3 * *$ & $\rightarrow 0,34 * *$ & - & $0,36^{* *}$ \\
\hline Maximum soir et Durée soir ........ & $-0,22 *$ & $-0,47^{* *}$ & $-0,42 * *$ & - & $0,40 * *$ \\
\hline Maximum total et Durée totale ..... & $-0,19 \mathrm{NS}$ & $-0,52 * *$ & $-0,29 * *$ & - & $0,37 * *$ \\
\hline
\end{tabular}

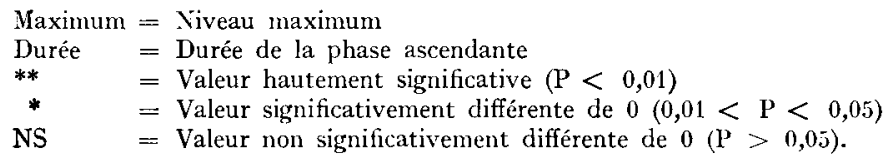

Les corrélations phénotypiques, calculées entre la production maximum et la production en 60 jours de lactation ou la production durant toute la lactation, sont supérieures à 0,73 pour chacun des 3 types d'animaux (tab1. 4). Boyazoglu, Poly et Poutous (1965), trouvent également, sur des lactations tronquées de brebis Lacaune, ne débutant en moyenne qu'au $35^{\mathrm{e}}$ jour de lactation, une forte corrélation phénotypique entre la production au contrôle maximum et la production totale $(+0,75)$. Plusieurs autres auteurs ont trouvé sur les Bovins comme sur les Ovins laitiers des valeurs aussi élevées (DECAEN et Poutous, I965). En outre, la corrélation génétique entre les 2 variables est estimée à $+0,95$ par Boyazoglu, Poly et Poutous (Ig65). 
Ces résultats soulignent l'intérêt du niveau maximum de production laitière comme estimation précoce des aptitudes laitières des brebis comme des vaches.

\section{TABLEAU 4}

Coefficients de corrélation et de régression entre la production maximum journalière, la production en 60 jours et la production totale

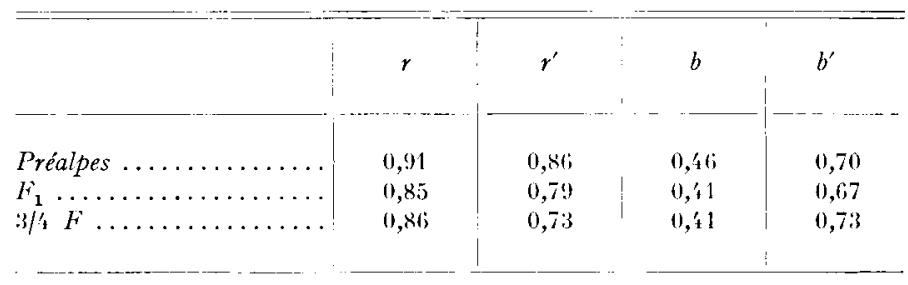

Valeurs toutes hautement significatives $(\mathrm{P}<0,01)$.

$r$ : corrélation entre production maximum journalicre et production en $60 \mathrm{j}$. $b$ : régression de la production en $60 \mathrm{j}$ sur la production maximum journalière. $r^{\prime}$ : corrélation entre production maximum journalière et production totale. $b^{\prime}$ : régression de la production totale sur la production naximum journalière.

Age et numéro de lactation.

Les productions maxima obtenues en première lactation à 2 ans sont nettement plus élevées que lors d'une première lactation à I an. La différence entre les 2 groupes de brebis persiste d'ailleurs au cours des lactations suivantes (fig. 2). Le niveau de

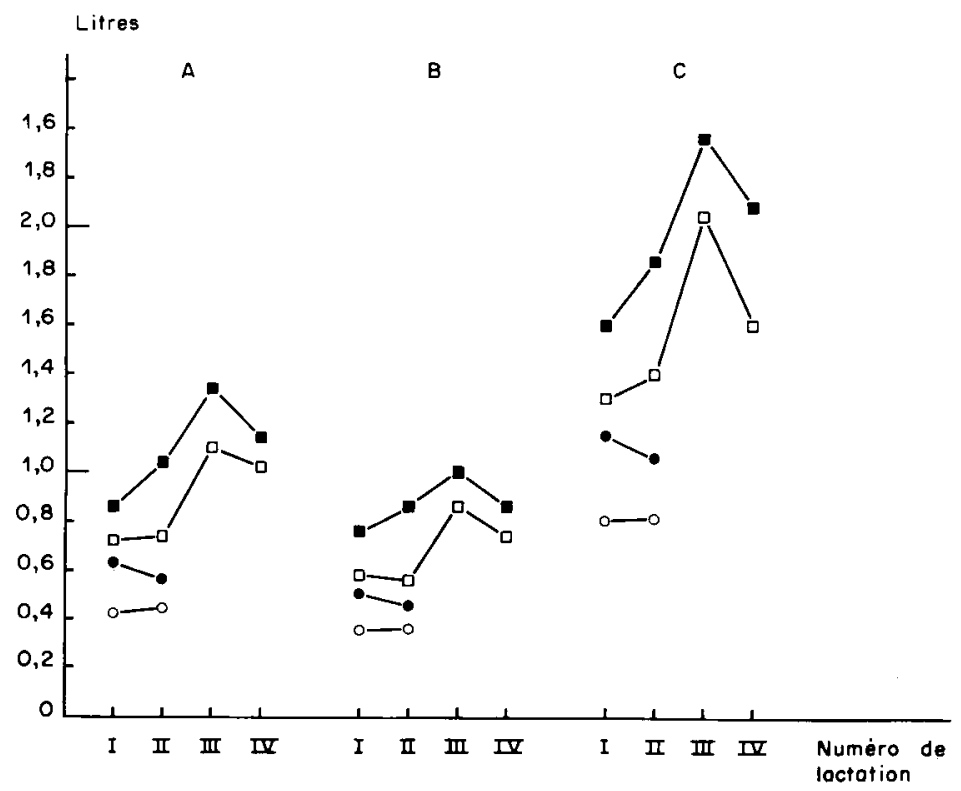

FIG. 2. - Productions moyennes maximales selon l'âge au premier agnelage

- Préalpes. Premier agnelage à I an - Préalpes. Premier agnelage à 2 ans

$\square \mathrm{F}_{1}$. Premier agnelage $\grave{\mathrm{a}} \mathrm{I}$ an $\quad \mathrm{F}_{1}$. Premier agnelage à 2 ans

A Production machine-matin $B$ Production machine-soir

$\mathrm{C}$ Production totale (machine + égouttage) 
production maximum augmente avec l'âge tout particulièrement pour les animaux $3 / 4 \mathrm{~F}$. Cependant, pour les animaux Préalpes et $\mathrm{F}_{1}$ ayant eu leur première lactation à $\mathrm{I}$ an, les différences entre $\mathrm{I}^{\mathrm{re}}$ et $2^{\mathrm{e}}$ lactation sont souvent très faibles (tab1. 5). Ceci s'explique par les besoins importants (gestation et lactation) imposés à partir de ro mois aux agnelles pendant leur propre période de croissance qui ne s'achève vrai-

Performances moyennes et durées de la phase ascendan

\begin{tabular}{|c|c|c|c|c|c|c|c|c|c|c|c|c|c|}
\hline \multirow{3}{*}{ Age } & \multirow{3}{*}{ Race } & \multicolumn{6}{|c|}{1962} & \multicolumn{6}{|c|}{1963} \\
\hline & & \multirow{2}{*}{$n$} & \multicolumn{2}{|c|}{$\begin{array}{l}\text { Froduction } \\
\text { maximum }\end{array}$} & \multicolumn{2}{|c|}{$\begin{array}{l}\text { Production } \\
\text { en } 60 \text { jours }\end{array}$} & \multirow{2}{*}{$\begin{array}{l}\text { Durée } \\
\text { jours }\end{array}$} & \multirow{2}{*}{$n$} & \multicolumn{2}{|c|}{$\begin{array}{l}\text { Production } \\
\text { maximum }\end{array}$} & \multicolumn{2}{|c|}{$\begin{array}{l}\text { Production } \\
\text { en } 60 \text { jours }\end{array}$} & \\
\hline & & & $\mathrm{ml}$ & p. 100 & 1 & p. 100 & & & $\mathrm{ml}$ & p. 100 & 1 & p. 100 & \\
\hline 1 an & $\begin{array}{c}\text { Préalpes } \\
\mathrm{F}_{1} \\
3 / 4 F\end{array}$ & $\begin{array}{l}21 \\
20 \\
-\end{array}$ & $\begin{array}{r}808 \\
1308 \\
-\end{array}$ & $\begin{array}{l}100 \\
162 \\
-\end{array}$ & $\begin{array}{l}42 \\
70 \\
-\end{array}$ & $\begin{array}{l}100 \\
167 \\
-\end{array}$ & $\begin{array}{l}34 \\
34 \\
-\end{array}$ & $\begin{array}{l}17 \\
23 \\
17\end{array}$ & $\begin{array}{r}669 \\
1329 \\
1348\end{array}$ & $\begin{array}{l}100 \\
199 \\
201\end{array}$ & $\begin{array}{l}31 \\
67 \\
64\end{array}$ & $\begin{array}{l}100 \\
216 \\
207\end{array}$ & $\begin{array}{l}31 \\
28 \\
20\end{array}$ \\
\hline \multicolumn{2}{|c|}{ Test races } & 41 & \multicolumn{2}{|c|}{$* *$} & \multicolumn{2}{|c|}{-} & NS & 57 & \multicolumn{2}{|c|}{$* *$} & \multicolumn{2}{|c|}{-} & NS \\
\hline 2 ans & $\begin{array}{c}\text { Préalpes } \\
\mathrm{F}_{1} \\
3 / 4 F\end{array}$ & $\begin{array}{l}\text { - } \\
\text { - }\end{array}$ & $\begin{array}{l}- \\
-\end{array}$ & - & - & - & E & $\begin{array}{l}17 \\
18 \\
-\end{array}$ & $\begin{array}{r}815 \\
1395 \\
-\end{array}$ & $\begin{array}{l}100 \\
171 \\
-\end{array}$ & $\begin{array}{l}42 \\
65 \\
-\end{array}$ & $\begin{array}{l}100 \\
155 \\
-\end{array}$ & $\begin{array}{l}21 \\
19\end{array}$ \\
\hline \multicolumn{2}{|c|}{ Test races } & - & & & & & - & 35 & \multicolumn{2}{|c|}{$* *$} & \multicolumn{2}{|c|}{ 一 } & NS \\
\hline 3 ans & $\begin{array}{c}\text { Préalpes } \\
\mathrm{F}_{1} \\
3 / 4 F\end{array}$ & E & $\begin{array}{l}- \\
-\end{array}$ & $\begin{array}{l}- \\
- \\
-\end{array}$ & $\begin{array}{l}- \\
- \\
-\end{array}$ & $\begin{array}{l}- \\
- \\
-\end{array}$ & 二 & $\begin{array}{l}- \\
-\end{array}$ & $\begin{array}{l}- \\
-\end{array}$ & $\begin{array}{l}- \\
- \\
-\end{array}$ & - & - & \\
\hline \multicolumn{2}{|c|}{ Test races } & - & \multicolumn{2}{|c|}{ - } & \multicolumn{2}{|c|}{ 一 } & 一 & - & \multicolumn{2}{|c|}{ - } & \multicolumn{2}{|c|}{ - } & \\
\hline $\begin{array}{l}\text { Test } \\
\text { âges }\end{array}$ & $\begin{array}{c}\text { Préalpes } \\
F_{1} \\
3 / 4 F\end{array}$ & 二 & \multicolumn{2}{|c|}{$\begin{array}{l}- \\
-\end{array}$} & \multicolumn{2}{|c|}{ - } & $=$ & $\begin{array}{l}34 \\
41 \\
-\end{array}$ & \multicolumn{2}{|c|}{$\begin{array}{l}\text { NS } \\
\text { NS } \\
-\end{array}$} & \multicolumn{2}{|c|}{$\begin{array}{l}- \\
-\end{array}$} & $* *$ \\
\hline
\end{tabular}

NS $=$ Différence non significative $(\mathrm{P}>0,05)$.

$*$ = Différence significative $(0,01<\mathrm{P}<0,05)$.

** $=$ Différence hautement significative $(\mathrm{P}<0,01)$.

ment qu'à 3 ou 4 ans (RICORDEAU et FI,AMAN'T, I969 b) ; il en résulte un retard dans le développement qui doit être comblé au cours de la deuxième lactation et limite ainsi le niveau des performances laitières. Pour les animaux $3 / 4 F$, les différences entre les 2 premières lactations sont nettes, une amélioration des conditions d'élevage du troupeau étant intervenue l'année de naissance de ces animaux ; mais ces brebis ont pu avoir également une meilleure précocité de développement due à leur pourcentage de sang Frison plus important (RICORDEAU et FlaMANT, Ig69 b). 
Année de production.

Le facteur " année " intervient sur le niveau de production maximum mais de façon variable selon les races et tout particulièrement si l'on considère les brebis qui mettent bas à I an. Ainsi, les productions moyennes des antenaises Préalpes, varient beaucoup plus d'une année à l'autre que celles des brebis croisées de même

\section{AAU 5}

Ion la race, l'âge et l'année de production

\begin{tabular}{|c|c|c|c|c|c|c|c|c|c|c|c|c|c|c|}
\hline \multicolumn{6}{|c|}{1964} & \multicolumn{6}{|c|}{1965} & \multicolumn{3}{|c|}{ Tests années } \\
\hline \multirow{2}{*}{$n$} & \multicolumn{2}{|c|}{$\begin{array}{l}\text { Production } \\
\text { maximum }\end{array}$} & \multicolumn{2}{|c|}{$\begin{array}{l}\text { Production } \\
\text { en } 60 \text { jours }\end{array}$} & \multirow{2}{*}{$\begin{array}{l}\text { Durée } \\
\text { jours }\end{array}$} & \multirow{2}{*}{$n$} & \multicolumn{2}{|c|}{$\begin{array}{l}\text { Production } \\
\text { maximum }\end{array}$} & \multicolumn{2}{|c|}{$\begin{array}{l}\text { Production } \\
\text { en } 60 \text { jours }\end{array}$} & \multirow{2}{*}{$\begin{array}{c}\text { Durée } \\
\text { jours }\end{array}$} & \multirow{2}{*}{$n$} & \multirow{2}{*}{$\begin{array}{l}\text { Prod. } \\
\text { max. }\end{array}$} & \multirow{2}{*}{ Durée } \\
\hline & $\mathrm{ml}$ & p. 100 & 1 & p. 100 & & & $\mathrm{ml}$ & p. 100 & I & p. 100 & & & & \\
\hline 20 & 810 & 100 & 36 & 100 & 30 & 17 & 567 & 100 & 25 & 100 & 25 & 75 & ** & NS \\
\hline 一 & - & - & - & - & - & - & - & - & - & -- & -. & 43 & NS & NS \\
\hline 29 & 1234 & 152 & 57 & 158 & 19 & 37 & 1231 & 217 & 56 & 226 & 26 & 83 & $\mathrm{NS}$ & $\mathrm{NS}$ \\
\hline 49 & \multicolumn{2}{|c|}{$* *$} & \multicolumn{2}{|c|}{-} & NS & $5 / t$ & \multicolumn{2}{|c|}{$* *$} & \multicolumn{2}{|c|}{ - } & NS & - & - & - \\
\hline 20 & 955 & 100 & 42 & 100 & 26 & 24 & 913 & 100 & 36 & 100 & 19 & 61 & NS & NS \\
\hline 21 & 1760 & $18 \dot{4}$ & 78 & 186 & 12 & - & - & 一 & - & - & - & 39 & $* *$ & $* *$ \\
\hline 12 & 1. 748 & 183 & 77 & 183 & $1 t^{\prime}$ & 26 & 1569 & 172 & 68 & 189 & 16 & 38 & $* *$ & NS \\
\hline 53 & \multicolumn{2}{|c|}{ ** } & \multicolumn{2}{|c|}{ - } & $* *$ & & \multicolumn{2}{|c|}{ ** } & \multicolumn{2}{|c|}{ - } & NS & - & - & - \\
\hline - & - & 一 & 二 & - & - & 21 & 1028 & 100 & 45 & 100 & 24 & - & - & - \\
\hline 14 & 2035 & 一 & 91 & - & 14 & 19 & 1941 & 189 & 88 & 196 & $1^{\prime} \mathrm{t}$ & 33 & ** & NS \\
\hline - & 一 & - & - & - & - & 08 & 1765 & 172 & 79 & 176 & 15 & - & - & - \\
\hline - & \multicolumn{2}{|c|}{-} & \multicolumn{2}{|c|}{-} & - & 48 & \multicolumn{2}{|c|}{$* *$} & \multicolumn{2}{|c|}{-} & $*$ & - & - & 一 \\
\hline 40 & \multirow{3}{*}{\multicolumn{2}{|c|}{$\begin{array}{l}\text { NS } \\
* * \\
* *\end{array}$}} & \multirow{2}{*}{\multicolumn{2}{|c|}{-}} & NS & 62 & \multicolumn{2}{|c|}{ ** } & \multicolumn{2}{|c|}{ 一 } & NS & - & - & 一 \\
\hline 35 & & & & & NS & - & \multirow{2}{*}{\multicolumn{2}{|c|}{$* *$}} & \multirow{2}{*}{\multicolumn{2}{|c|}{ - }} & - & - & 一 & - \\
\hline 51 & & & & - & NS & 71 & & & & & $* *$ & - & - & - \\
\hline
\end{tabular}

âge. Pour les années I963 et I965, les brebis croisées manifestent une supériorité de 210 p. Ioo par rapport aux Préalpes, contre I57 p. I00 en I962 et I964, alors que le niveau moyen de production des brebis croisées a peu varié au cours de ces 4 années (tabl. 5). Ce comportement différent s'est traduit dans les analyses statistiques par des interactions années-races hautement significatives. Comme nous l'avons montré précédemment (RICORDEAU et FLAMANT, I969 b), il semble que les animaux issus du croisement soient de développement plus précoce, ce qui les rend moins sensibles à des variations de conditions d'élevage notamment dans le cas d'une première lactation à I an. 
Race.

L'utilisation de béliers Frisons sur les brebis Préalpes apporte un accroissement de la production maximum journalière hautement significatif et de l'ordre de 80 à $85 \mathrm{p}$ IOo, résultat qui recoupe nos observations antérieures (RICORDEAU et FLAMANT, $\mathrm{I} 969 \mathrm{~b}$ ). Nous constatons, là aussi, que les écarts entre $\mathrm{F}_{1}$ et animaux possédant plus de 50 p. Ioo de sang Frison ne sont pas significatifs. Remarquons également que la supériorité des animaux croisés est légèrement plus élevée (4 à 6 p. Ioo) pour la production en 60 jours que pour le maximum journalier, ce qui traduit vraisemblablement leur meilleure persistance au-delà du maximum (tabl. 5).

Brebis.

Le facteur brebis intra-race, estimé à partir des éléments de l'analyse de variance hiérarchique, apparaît hautement significatif pour le niveau de production maximum chez les animaux de race pure Préalpes $d u$ Sud, peu significatif chez les $\mathbf{F}_{\mathbf{1}}$ et non significatifs chez les $3 / 4 \mathrm{~F}$.

Le coefficient de répétabilité calculé après correction des variables pour le numéro de lactation est généralement de même ordre pour les 3 variables de production considérées à l'intérieur d'une même race. Pour les différentes races, les valeurs obtenues sont légèrement plus faibles que celles habituellement ađmises pour la production totale (Préalpes : $r=+0,52 \mathrm{P}<0, \mathrm{OI} ; \mathrm{F}_{1}: r=+0,35 \mathrm{P}<0, \mathrm{OI} ; 3 / 4 F: r+0,32$ 0 ,OI $<\mathrm{P}<0,05)$.

\section{Facteurs de variation de la durée de la phase ascendante}

La corrélation entre les durées de la phase ascendante matin, soir et total est beaucoup plus faible que pour la quantité maximum, surtout entre durée-matin et durée-soir $(0,38$ à 0,85 ; tab1. 3$)$, par suite vraisemblablement de l'imprécision avec laquelle on détermine la date de production maximum. Néanmoins, les résultats obtenus au cours des analyses sont semblables pour les 3 variables considérées.

\section{Age et numéro de lactation.}

La durée de la phase ascendante est généralement plus longue pour les animaux ayant eu leur première lactation à $I$ an. Cependant, les différences sont très faibles ( $I$ à 6 jours, fig. 3) et sont beaucoup moins importantes que les différences entre numéros de lactation : le maximum de production est en effet atteint nettement plus tardivement ( 5 à Io jours) en première lactation que lors des lactations suivantes (tabl. 5) quel que soit l'âge à la première mise bas.

\section{Année de production.}

Contrairement à ce qui est observé pour la production maximum, 1'année de production u'a pas d'influence sur la durée de la phase ascendante (tabl. 5).

Race.

Le maximum de la lactation apparaît en moyenne le $25^{\mathrm{e}}$ jour pour les $\mathrm{I} 26$ brebis croisées $\left(\mathrm{F}_{1}\right.$ et $\left.3 / 4 F\right)$ de $\mathrm{I}$ an et le $\mathrm{I}_{5}$ e jour pour les II 8 de 2 et 3 ans; pour les 
75 Préalpes de $\mathrm{x}$ an et les 82 de 2 et 3 ans, il se manifeste respectivement le $30^{\mathrm{e}}$ et le $22^{\mathrm{e}}$ jour. Ces écarts entre races atteignent quelquefois le seuil de signification statistique; résultat confirmé par l'analyse de variance hiérarchique réalisée sur toutes les données. Notons que la situation du maximum trouvé sur les Préalpes correspond bien à celui observé antérieurement (25 jour de lactation) sur 126 lactations de brebis de tous âges par RICORDEAU et DENAMUR (I962).

Brebis.

Les coefficients de corrélation intra-brebis de la durée de la phase ascendante calculés pour chaque race ne sont pas significativement différents de o (respectivement $0,09,0,06$ et $0, \mathrm{I} 7$ pour Préalpes, $\mathrm{F}_{1}$ et $3 / 4 F$ ).

A

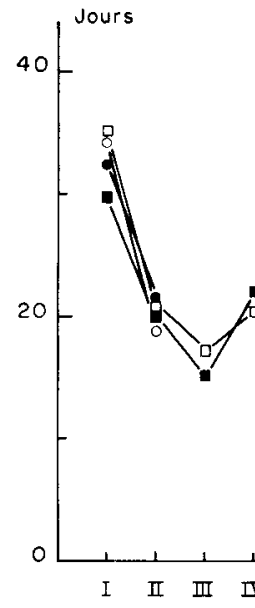

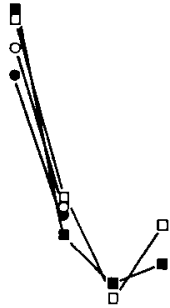

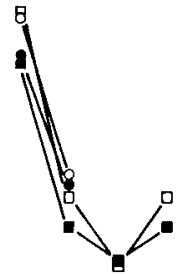

Frc. 3. - Durées moyennes de la phase ascendante de la lactation selon l'áge au premier agnelage

- Préalpes. Premier agnelage à $\mathbf{I}$ an

- Préalpes. Premier agnelage à 2 ans

- $\mathrm{F}_{1}$. Premier agnelage à $\mathrm{I}$ an

- $\mathrm{F}_{1}$. Premier agnelage à 2 ans

A Machine-matin

B Machine-soir

C Total (machine + égouttage)

Niveau de production.

La corrélation entre le niveau de production maximum et la durée de la phase ascendante est toujours négative $(-0,29$ à $-0,79$, tab1. 3). Une correction des données pour le numéro de lactation conduit à des valeurs plus faibles mais toujours négatives $\left(r=-0,20\right.$ et $-0,25$ pour les $\mathrm{F}_{1}$ et $\left.3 / 4 F\right)$ ou non différentes de 0 $(r=-0,04$ pour les Préalpes).

\section{DISCUSSION}

Facteurs de variation de la durée de la phase ascendante

I,e numéro de lactation et la race des brebis constituent les 2 principaux facteurs de variation de la durée de la phase ascendante de la lactation. Sur les bovins lai- 
tiers, DeCakn et Poutous (I965) constatent également que le maximum de production apparaît plus précocement en deuxième lactation. Ces effets doivent être interprétés différemment selon la signification biologique accordée au stađe d'apparition du maximum de la lactation.

L'évolution de la production laitière au cours de la lactation reflétant 1'importance du tissu mammaire et son activité sécrétrice, la phase ascendante de la lactation caractérise donc la période d'activation de la mamelle. D'après nos résultats, celle-ci atteindrait sa capacité maximum de production plus tardivement en première lactation, le tissu mammaire étant à un niveau de développement inférieur à celui du début des lactations ultérieures. En outre, les brebis croisées manifesteraient pour ce caractère une meilleure précocité, déjà constatée par ailleurs pour d'autres critères (RICORDEAU et FlamANT, Ig69 $b$ ).

La phase ascendante de la lactation peut également traduire l'intervention de facteurs limitants : absence du réflexe d'éjection du lait lors de la traite et insuffisance des apports alimentaires.

Une phase ascendante courte peut donc refléter une adaptation rapide à la traite. Les effets inhibiteurs, liés dans notre cas particulier à une traite mécanique dès les premiers jours après la mise bas, seraient alors moins importants en deuxième lactation qu'au début de la première lactation quel que soit l'âge des brebis. Par ailleurs, les brebis croisées manifesteraient de ce point de vue une meilleure facilité de traite bien que RICORDEAU et FIAMANT $(\operatorname{Ig} 69 b$ ) aient montré que ces animaux ont un volume d'égouttage plus important.

En ce qui concerne l'influence du niveau d'alimentation, la discussion est plus difficile. Il est certain que les apports alimentaires sont presque toujours inférieurs aux besoins des bonnes laitières, tout particulièrement en début de lactation, et supérieurs à ceux des médiocres productrices. Nous sommes donc amenés à envisager les relations entre le volume du maximum de la lactation et sa position par rapport à la mise bas. Ricordeau et Denamur (I962), PEART (I967), observent sur des brebis allaitantes que les animaux avec deux agneaux atteignent leur maximum de production quelques jours plus tôt que les brebis avec un seul agneau. Mais ce résultat peut s'expliquer par le fait que les jumeaux tètent plus fréquemment lorsqu'ils sont jeunes et lorsqu'ils se nourrissent exclusivement de lait. D'ailleurs, en considérant une même catégorie de brebis, RicoRdEAU et DENAmur ne trouvent pas de liaison entre le niveau maximum et le stade auquel il intervient. STARKE (r953) et HunTER (1956) arrivent à la même conclusion. D'autres auteurs, considérant toujours des brebis allaitantes, estiment que les médiocres productrices atteignent le maximum plus tôt quelle que soit, semble-t-il, l'origine des différences de production (génétique ou conditions de milieu). C'est le cas de Bonsma (I939), WALLACF (I948), BARNicoAT, LogaN et GRANT (I949). Il s'agit là en fait de résultats observés par pesée des agneaux avant et après tétée, à la suite de contrôles effectués toutes les semaines.

Sur des brebis Préalpes traites depuis le début de la lactation, RicordeaU et Denamur (I969) obtiennent comme nous une liaison négative ( $\mathrm{r}=-0,39$ ) entre le niveau de production maximum et la durée de la phase ascendante et remarquent en particulier que le maximum de production des meilleures laitières est très prononcé et atteint précocement.

Chez les Bovins laitiers, les résultats sont également contradictoires. MAYMone et Maiossini (I96I), Decaen et Poutous (1965) obtiennent une liaison négative, 
tandis que Turner, Ragsdale et Brody (I923) et Journeit et JARrige (I960) enregistrent le contraire. Dans tous les cas, les valeurs trouvées sont très faibles. Pour comparer valablement ces résultats, il faudrait tenir compte du programme de rationnement avant et après le vêlage. On peut en effet admettre qu'avec une alimentation individuelle en fonction du niveau de production, les meilleures laitières tendent à atteindre leur maximum de production plus tardivement par suite des ajustements successifs de rations. Par contre, dans les troupeaux ovins laitiers, où le rationnement est effectué globalement pour tous les animaux en vue d'assurer des besoins de production moyens, les meilleures laitières sont limitées dès le début de la production : ce type de conduite du troupeau expliquerait donc aussi pourquoi les maxima de production, plus élevés en deuxième lactation et avec les brebis croisées, sont atteints plus précocement.

\section{Caractéristiques des distributions de fréquence}

La dissymétrie des distributions de la durée de la phase ascendante de la lactation a été également observée sur la vache par DRAKELEY et WHITE (I927), MAYmone et Mai,ossini (I959-I96I), RAKEs et al. (I959), MAKela (I962 b) et DECAEN et Pourous ( 1965 ). Ces auteurs trouvent une position moyenne du maximum de lactation entre le $25^{\mathrm{e}}$ et le $40^{\mathrm{e}}$ jour, soit de une à deux semaines plus tard environ que chez la brebis d'après nos résultats. Quant au mode, il se situe entre le roe et le I $5^{\mathrm{e}}$ jour, c'est-à-dire à peu près dans les limites observées dans notre étude. DECAEN et Poutous (1965) trouvent un coefficient de variation légèrement supérieur au nôtre ( $75 \mathrm{p}$. Ioo contre $60 \mathrm{p}$. IOO), valeur élevée qu'ils attribuent également à l'existence de maxima de lactation très tardifs (jusqu'à 80 jours).

Peut-on donner une explication de cette dissymétrie, compte tenu des ajustements possibles à des distributions de fréquence théoriques ? MAYMONE et MALOSSINI (I96I) parviennent, chez la vache, à une distribution normale en réalisant une transformation logarithmique des données. Dans notre cas, cette transformation augmente encore les écarts par rapport à une distribution normale. Par contre, la transformation racine carrée rend toutes les distributions symétriques, y compris les distributions de la quantité maximum ; des écarts avec la loi normale subsistent cependant pour l'indice d'aplatissement (tabl. 2). Ces résultats indiquent une certaine proportionnalité entre la moyenne et l'écart-type des distributions. Nous avons testé l'ajustement à une distribution de Poisson par le test du $\chi^{2}$ en utilisant comme variable non pas le jour d'apparition des maxima de production, mais la semaine. Les écarts à une distribution de Poisson ne sont pas significatifs pour les données Préalpes et $\mathrm{F}_{1}$, mais l'ajustement n'est pas possible avec les $3 / 4 F$ qui ne constituent d'ailleurs pas un groupe homogène.

Si nous retenons l'hypothèse d'une distribution de Poisson, nous pouvons alors considérer que dans une race donnée, la semaine d'apparition du maximum de lactation d'une brebis, variable bornée inférieurement à o mais non supérieure nent, s'applique à un événement de fajble probabilité lié à un certain nombre de facteurs de variation. Il n'est donc pas surprenant que nous ayons trouvé, pour la durée de la phase ascendante, des coefficients de répétabilité non différents de 0 .

La dissymétrie constatée peut résulter également de l'existence de 2 sous-populations de moyennes et de variances différentes. C'est ainsi que LABUSSrÈRE (I966) 
a mis en évidence, dans le même troupeau, deux catégories de brebis : celles qui présentent une seule émission de lait au cours de la traite ont un maximum de production peu élevé et précoce, alors que les brebis à " 2 émissions " ont, en moyenne, un maximum très prononcé et tardif. La relation décelée par LABUSSIÈrRE entre les 2 types de descente du lait au cours de la traite et le niveau de production pourrait donc expliquer la dissymétrie trouvée dans la durée de la phase ascendante et le niveau de production maximum; mais cela suppose une corrélation positive entre les 2 variables alors que nous avons obtenu une corrélation négative. D'autre part, la caractéristique de traite (I ou 2 émissions) est un caractère relativement constant d'une lactation à l'autre alors que les variables considérées sont peu répétables. La validité de cette tentative d'explication de la forme des distributions par des considérations physiologiques est limitée par le fait qu'en plus des brebis à I et 2 émissions, il existe d'autres brebis, bonnes laitières dans l'ensemble, qui ne peuvent être classées nettement dans l'une des deux catégories.

\section{CONCLUSION}

Ce travail est encore incomplet car il ne tient pas compte de l'évolution de la production depuis le premier jour de traite, et de sa variation de part et d'autre du maximum. D'autre part, les brebis étant alimentées par lots, il n'a pas été passible de comparer le niveau d'alimentation individuel avec le niveau maximum. Cette étude a permis cependant de compléter les observations déjà réalisées sur la supériorité des brebis croisées Frisonnes par rapport aux Préalpes pour la production laitière, la persistance de la lactation et la précocité de développement. Nous avons pu aussi mettre en évidence l'influence du numéro de lactation et de la race sur la durée de la phase ascendante. Iais il est difficile, faute d'observations plus fines au niveau de la mamelle (activité du tissu sécrétoire, courbes de descente de lait), de déterminer parfaitement les causes biologiques des différences observées entre première lactation et lactations ultérieures, entre brebis Préalpes et croisées : il est vraisemblable que le niveau d'alimentation constitue un facteur de variation en liaison avec l'établissement de l'activité sécrétrice et l'adaptation à la traite. Une difficulté supplémentaire apparaît, d'ordre statistique celle-ci, du fait de la nature des distributions des variables considérées : comme chez les Bovins, l'ajustement à une distribution normale ne peut être envisagé même après diverses transformations. Dans notre cas, la transformation racine carrée serait cependant susceptible de donner de bons résultats.

L'ensemble des observations réalisées au cours de cette étude ne sont pas différentes de celles effectuées par d'autres auteurs sur les Bovins : sur le plan alimentaire, dans les 2 espèces, l'accroissement des besoins est considérable de la fin de la gestation aux premières semaines de la lactation et doit être mis en comparaison avec l'évolution de l'appétit des animaux durant la même période. Toutefois, chez les Ovins traditionnellement soumis à la traite, il faut envisager le cas particulier de la séparation des agneaux après 30 à 40 jours d'allaitement. Nos résultats montrent que le maximum de la lactation se situe à la même période pour les brebis allaitantes et les brebis traites : 65 à $80 \mathrm{p}$. Ioo des lactations selon les races ont atteint leur maximum avant la $5^{\mathrm{e}}$ semaine, c'est-à-dire avant le sevrage. En con- 
sidérant également les travaux de LABUssière et PETREQuiN (I969), nous constatons que pour les "brebis laitières ", le passage de l'allaitement à la traite constitue un stade critique sur le plan physiologique de la sécrétion et de l'éjection du lait, au moment du maximum d'activité de la mamelle.

Ręu pour publication en mai 1969.

\section{REMERCIEMENTS}

Nous tenons à remercier MM. Boyazoglu, Denamur, JARrige et Vissac pour les conseils qu'ils nous ont donnés au cours de la rédaction de ce mémoire.

\section{SUMMARY}

\section{PRELIMINARY STUDY OF THE ASCENDING PHASE \\ OF THE LACTATION CURVE IN MILKING EWES}

We studied the maximum lactation stage in 544 lactations in a flock of pure-bred Préalpes $d u$ Sud and Friesland cross ewes. The respective performances of pure-bred and crossed ewes been previously studied (RICORDEAU and FLAMANT, Ig69 $a$ and $b$ ).

The frequency distributions of the ascending lactation phase are very dissymetrical (table I, fig. I), and logarithmic transformation does not permit an adjustement to the normal distribution. "This adjustement may be partially accomplished by a square root transformation. The observed dissymetry may occur, because the week when maximum lactation appears is a variable having a lower limit of zero, but no upper limit, and it is applied to an occurence of weak probability.

The main variation factors observed are the lactation number and breed. Those animals in second or third lactations, no matter what their age, have an earlier maximum lactation than during the first lactation (5 to ro days). Maximum lactation appears on an average on day 25 in one-year old crossed ewes, and on day i 5 in 2-3 year old crossed ewes. The corresponding days in the Préalpes du Sud breed are the thirtieth and twenty-second days (table 5). After correction for the lactation number, the correlation between the length of the ascending phase and production level (computed for each breed) remains weak and negative (-0.04 to -0.25$)$.

\section{RÉFÉRENCES BIBLIOGRAPHIQUES}

Barnicont C. R., Logan A. G., Grant A. I., i 949. Milk secretion studies with New Zealand Romney ewes. J. agric. Sci., 39, 44-237.

Bonsma F. X., 1939. Factors influencing growth and development of lambs, with special reference to cross-breeding of Merino sheep for fat lamb production in South Africa. Publ. Univ. Preloria Agric., 48, $214 \mathrm{p}$.

Boyazoglu J., Catrin-Vidal P., i 964. Aspects quantitatifs de la production laitière des brebis. Bull. Tech. Int., 191, $455^{-470}$.

Boyazogly J., Poly J., Poutous M., ic,65. Aspects quantitatifs de la production laitière des brebis. IV. Corrélations génétiques et phénotypiques. Ann. Zootech., 14, I45-152.

Decaen C., Pottot's M., 1965. Phase ascendante de la courbe de lactation chez la vache laitière. Ann. Zootech., 14, 135-153.

DRAKELEY T. S., WhiTl: M. K., I927. The infuence of the stade of lactation and the breeds of the cows on the yield and quality of milk. J. agric. Sci., 17, rog.

Hunter G. L., 1956. The maternal influence on size in sheep. J.agrit. Sci., Camb., 48, 36-60.

Journet M., JARRIGE R., I960. Évolution de la sécrétion des matières gras ses, des matières azotées et du lactose au cours du premier mois de lactation. Ann. Zootech., 9, I33-I35.

LABUSSIERE J., I.966. Relation entre le niveau de production laitière des brebis et leur aptitude à la traite XVII. Int. Milchwirtschaftskongr. Band A., 43-5 I.

Labussilere J. Petrequix P., i 969 . Relation entre l'aptitude à la traite des brebis et la perte de production laitière constatée au moment du sevrage. Ann. Zootech., 18 (1), 5-15. 
MAKEL A A., I962. The lactation curve of the cow at the ascending phase. Maalalousl. Aikakausk., 34, I 73186.

Maymone, B., Malossini F., 1959. The rising phase of the lactation curve in dairy cows. Z. Tierzucht. Ziichl Biol., 78, 276-294.

Maymone B., Malossins F., ig6r. La curva di lattazione della bufale. Alimentaz. anim., 1, i 9-40.

PEarson E. S., Hartley H. O., I958. Biometrika tables for slatisticians. Vol. I. Cambridge University Press, 240 p.

PEART J. N., I 967. The effect of different levels of nutrition during late pregnancy on the subsequent milk production of Black face ewes and on the growth of their lambs. J. agric. Sci., Camb., 68, 365-37 I.

Rakes J. M., Stallcup O. T., Horton O. H., Gifrord W., 1959. Maximum daily milk production as affected by certain factors. Agric. Exp. Sin., Univ. Arkans. Bull. 6 r 5 .

Ricordeau G., Denamur, R., 1962. Production laitière des brebis Préalpes du Sud pendant les phases d'allaitement, de sevrage et de traite. Ann. Zootech., 11, 5-38.

Ricordeau G., Flamant J. C., ig69a. Croisements entre les races ovines Préalpes du Sud et Frisonne (Ostfrie'isches Milchschaf). II. Reproduction, croissance, conformation et viabilité. Ann. Zoolech., 18 (2), I 5 I-I 68.

Ricordeau Cr., Flamant J. C., 1968 b. Croisement entre les races ovines Préalpes du Sud et Frisonne (Ostfriesisches Milchschaf). III. Performances laitieres. Ann. Zootech., 18 (2), I31-149.

Starke J. S., 1953. Studies on the inheritance of milk production in sheep. S. Afr. J. anim. Sci., 49, 245-254.

Turner C. W., Ragsdale A. C., Brody S., ig23. How the advance of the period of lactation affects the milk flow. J. Dairy Sci., 6, 527-531.

WALLACE L. R., 1948. The growth of lambs before and after birth in relation to the level of nutrition. J. agric. Sci., 38, 93-I 53 . 